\title{
Preferred Mode of Delivery in Iraqi Primiparous Women
}

\author{
Farah Abdulhussein Salih Al-asadi', Abdullah Omar Ahmad ${ }^{2}$, \\ Rawan Jassem Mohammed ${ }^{2}$, Zeena Mohammed Noor Mohammed Maasom² \\ ${ }^{1}$ M.B.Ch.B, F.I.C.O.G, Lecturer at College of Medicine/University of Baghdad, \\ ${ }^{2} 4^{\text {th }}$ Grade Students at College of Medicine/University of Baghdad
}

\begin{abstract}
Background: Pregnancy is a physiological action that ends with pain, fear, anxiety and fear of death for the mothers. Delivery is a multi-dimensional process associated with physical, emotional, social, physiological, cultural and psychological dimensions.

Objectives: To identify the preferred mode of delivery in Iraq primiparous women and factors make them chose this mode of delivery.

Methodology: a descriptive/analytical cross-sectional study performed among 150 Iraqi primiparous women who attended the gynecology clinic in Baghdad teaching hospital/medical city from July to November 2019 the information regarding socio-demographical data and asking the women about the causes of this choice.

Results: Total of 150 primiparous women was enrolled in the study. Majority of them preferred vaginal delivery $(68.7 \%)$ while women who preferred cesarean section were $(31.3 \%)$. high rate of vaginal delivery preference was seen in women because of faster recovery, easy baby bonding, fear from cesarean section and short hospital stay, while those who preferred cesarean section thought that they can avoid labor pain and fear on baby's life.

Conclusion: Majority of primiparous women interviewed in our research preferred the vaginal delivery, while the remaining women preferred the cesarean section due to lack of knowledge or fear from vaginal delivery. Doctor's recommendation and medical advices play a major role in enhancing the awareness toward vaginal delivery.
\end{abstract}

Keywords: Primiparous, vaginal delivery, cesarean section, preferred mode of delivery.

\section{Introduction}

Pregnancy is a physiological action that ends with pain, fear, anxiety and fear of death for the mothers. Delivery is a multi-dimensional process associated with physical, emotional, social, physiological, cultural and psychological dimensions. ${ }^{[1]}$

The decision of mode of delivery is explained

\section{Corresponding Author:}

\section{Dr. Farah Abdul Hussein Salih Al-Asadi}

M.B.Ch.B, F.I.C.O.G, Lecturer at College of Medicine/ University of Baghdad

e-mail: farah_alasadya@yahoo.co.uk

Phone Number: +9647818069544 as preferring either the vaginal or cesarean section delivery. ${ }^{[2]}$ It's important for the pregnant woman to choose between the two modes of delivery. Some factors will effect on this decision like friends, media, health professionals, effective antenatal, fear of childbirth, the balance between the desires of the mother, the risks of a repeat operation, the risks to her child during labor and the risk of labor on the strength of the old scar. ${ }^{[3-5]}$

Childbirth can be divided into 'vaginal delivery' which is a complex interaction between the uterine contractions, the bony pelvis, the soft tissues of the pelvic floor and perineum and the fetus. Contractions are important to start the dilatation of the uterine cervix and descent of the fetus. ${ }^{[5]}$

Each pregnancy and delivery are different, and 
problems may arise with vaginal delivery are Perinatal asphyxia, Shoulder dystocia, Perineal tears, Excessive bleeding, abnormal fetal heart rate and premature rupture of membranes (PROM). ${ }^{[6-12]}$

The advantages of vaginal delivery are short hospital stay, faster recovery, and minimal blood loss so the possibility of bleeding, infection and mortality are less. Whereas the disadvantages are prolonged, painful, stressful labor, urinary incontinence, $4 \%$ of women giving birth shows fecal incontinence as a serious problem. ${ }^{[13-16]}$

'Cesarean section' is two steps of surgical intervention. Initially an incision is made through the mother's abdomen (laparotomy) after that another one is done to the uterus (hysterotomy) to deliver the fetus.

\section{Method}

Study design and participants: This descriptive/ analytical research was designed as a cross-sectional study. The setting was composed of all primiparous women who visited the gynecology clinic in Baghdad teaching hospital/medical city. The sample size that we collected from the pregnant women that visit antenatal care (ANC) was 150. The sampling method was a convenience sample. The inclusion criteria were primiparous women in the first, second, and third trimester who agreed to participate after we explained to them the purpose of this study. The exclusion criteria were the multiparous women and the women who had communication and participation error and who disagreed to participate in the study. This study was approved by College of medicine/University of Baghdad from July to November 2019.

Instruments: The data were collected by a researcher-designed questionnaire and was composed of two sections. The first section contained the preferred mode of delivery and the reason make them chose this mode of delivery [contains vaginal delivery and its three reasons (easy baby bonding, short hospital stay, faster recovery and other reasons) and contain cesarean section and its three reasons (avoid labor pain, fear on baby, can be scheduled and other reasons)]. The second section has 13 items include:

1. Name of participant.

2. Age of the pregnant woman: We divided the age into three intervals (The first interval is from the age of 15 to 25 , the second interval is from the age of 26 to 35 and the third interval is from the age of 36 to $45)$.

3. Age of husband: We divided the age of husband into four intervals (The first interval is from the age of 15 to 25 , the second interval is from the age of 26 to 35 , the third interval is from the age of 36 to 45 and the fourth interval is from the age of 46 to 55)

4. Residency.

5. Occupation of participant.

6. Level of education: We divided the level of education into five items (Illiterate, primary, secondary, college student and post- graduate(

7. Occupation of husband.

8. Period of infertility: (We considered that the women has a period of infertility if her period of infertility is 1 year and above, so any period below 1 year, we considered it as no infertility).

9. Gender of fetus if known.

10. Gestational age at time of enquiry: We divided the gestational age into three trimesters (The first trimester is from 1 week to 16 week, the second trimester is from 16 week to 28 week, and the third trimester is from 28 week to 40 weeks (

11. Previous surgery.

12. Bad previous family experience with cesarean section or vaginal delivery.

13. How many doctors she has follow.

In order to be sure about the validity of the data, various steps were taken as follows: long-term involvement with the participants during data collection; ongoing observations; trying different method of data collection and providing in depth descriptions.

Ethical Concept: Before we started collecting the data, approval was obtained from the Local Medical Research Ethics Committee, the hospital where the research was conducted and verbal approval was taken from each participant after we explained the purpose of the questionnaire.

Limitation: One of the limitations of the study is that it can't be generalized to all primiparous women because it was done in a single hospital in Baghdad and the second one is the small sample size.

Statistical Analysis: SPSS version 23 (Statistical 
Package for the Social Sciences) used for data entry and data analysis. Results presented in the form of tables and graphs. Chi square test used to assess association between descriptive data and Fisher exact test used if the chi square test is not applicable. $\mathrm{P}$ value $<0.05$ will be considered significant.

\section{Results}

In total 150 primiparous women complete the questionnaire. As shown in table 1 the vaginal delivery was presented in $(68.7 \%)$ and $\mathrm{Cs}$ in $(31.3 \%)$. The main reason for preferring $\mathrm{Cs}$ was to avoid labor pain (13.3\%) and the lesser cause was can be schedules (1.3\%), while main reason for preferring vaginal delivery was faster recovery $(38 \%)$ all these were found in table 1.

Table 2 shows the relationship between the number of doctors she has follow and preferred mode of delivery. no association between the number of doctors she has follow and preferred mode of delivery.
Table 1: Preferred and reason of mode of delivery

\begin{tabular}{|c|c|c|}
\hline & Frequency & Percent \\
\hline Vaginal delivery & 103 & $68.7 \%$ \\
\hline Cesarean section & 47 & $31.3 \%$ \\
\hline Total & 150 & $100 \%$ \\
\hline \multicolumn{3}{|l|}{ Reasons for preferring Cs } \\
\hline To avoid labor pain & 20 & $13.3 \%$ \\
\hline Fear on baby & 17 & $11.3 \%$ \\
\hline Fear from vaginal delivery & 5 & $3.3 \%$ \\
\hline Can be scheduled & 2 & $1.3 \%$ \\
\hline Other reasons & 3 & $2 \%$ \\
\hline Total & 150 & $100 \%$ \\
\hline \multicolumn{3}{|c|}{ Reason for preferring vaginal delivery } \\
\hline Faster recovery & 57 & $38 \%$ \\
\hline Easy baby bonding & 15 & $10 \%$ \\
\hline Fear from Cs & 9 & $6 \%$ \\
\hline Easy baby bonding, faster recovery & 7 & $4.7 \%$ \\
\hline Short hospital stays & 7 & $4.7 \%$ \\
\hline Short hospital stays, faster recovery & 4 & $2.7 \%$ \\
\hline Other reason & 4 & $2.7 \%$ \\
\hline Total & 4 & $100 \%$ \\
\hline
\end{tabular}

Table 2: How many doctors she has follow * Preferred mode of delivery Crosstabulation

\begin{tabular}{|c|c|c|c|c|c|}
\hline & & \multicolumn{2}{|c|}{ Preferred mode of delivery } & \multirow{2}{*}{ Total } & \multirow{2}{*}{ P Value } \\
\hline & & Cesarean section & Vaginal delivery & & \\
\hline \multirow{4}{*}{ How many doctors she has follow } & 1 Doctor & 30 & 65 & 95 & \multirow{4}{*}{0.643} \\
\hline & 2 Doctors & 7 & 19 & 26 & \\
\hline & 3 Doctors & 8 & 16 & 24 & \\
\hline & $<3$ doctors & 2 & 3 & 5 & \\
\hline \multicolumn{2}{|l|}{ Total } & 47 & 103 & 150 & \\
\hline
\end{tabular}

Table 3 shows the relationship between the bad previous family experience with cesarean section or vaginal delivery and preferred mode of delivery.

Because the minimum expected count is less than 5 , the chi square test is not applicable, so we reject the result of chi square and apply another test called fisher's exact test and according to this test the p-value is more than 0.05 , so we found no association between the bad previous family experience with cesarean section or vaginal delivery and preferred mode of delivery. 
Table 3: Bad previous family experience with cesarean section or vaginal delivery * Preferred mode of delivery Crosstabulation

\begin{tabular}{|c|c|c|c|c|c|}
\hline & & \multicolumn{2}{|c|}{ Preferred mode of delivery } & \multirow{2}{*}{ Total } & \multirow{2}{*}{ P Value } \\
\hline & & Cesarean section & Vaginal delivery & & \\
\hline \multirow{2}{*}{$\begin{array}{l}\text { Bad previous family experience with } \\
\text { cesarean section or vaginal delivery }\end{array}$} & No & 42 & 96 & 138 & \multirow{2}{*}{0.518} \\
\hline & Yes & 5 & 7 & 12 & \\
\hline \multicolumn{2}{|l|}{ Total } & 47 & 103 & 150 & \\
\hline
\end{tabular}

\section{Discussion}

Women's preferences for mode of delivery can differ according to their expectations and experiences. Some women are afraid about the health or the survival of baby during pregnancy or childbirth. So due to these reasons, it is important to respect the wishes of the primiparous women. ${ }^{[17]}$ Recent study showed that in developing countries, lower education level is the cause of poorer knowledge of human reproduction than women in developed countries. ${ }^{[18]}$

This study is about preferred mode of delivery in Iraqi primiparous women and factors that influence their decision. Data were analyzed to find association of certain demographic factors and their lifestyle with the decision-making.

The results of this study showed that majority of primiparous women preferred vaginal delivery in a percentage of $(68.7 \%)$. Similar to our study finding, another study conducted in Turkey, vaginal delivery rates are high in a percentage of $(87.7 \%)$. [19] Also similar to other study in Iran, vaginal delivery is higher than cesarean section in a percentage of $(80.4 \%)$. ${ }^{[20]}$

Another study performed by Ryding, Lukasse, and Krisjansdottir in six countries found that the primiparous women in these countries (Germany, Iceland, Denmark, Estonia, Norway, Sweden) preferred VD (85.1\%), (61.7\%), (77.5\%), (60.0\%), (71.1\%), (71.1\%), respectively. ${ }^{[21]}$

Primiparous women in our study prefer vaginal delivery due to faster recovery, easy baby bonding, fear from cesarean section, short hospital stay, better for health and less complication. Some of these reasons are similar to previous studies. ${ }^{[19]}$

On the other hand, the percentage of primiparous women who preferred caesarean section were (31.3\%). Similar to our study finding, in turkey the rate of pregnant women who preferred cesarean section was (7.6\%) similar findings were obtained from other countries the percentage in Singapore (3.7\%) and Sweden (8.7\%). ${ }^{[19]}$

Primiparous women in this study preferred cesarean section to avoid labor pain, fear on baby, it can be scheduled and fear on herself some of these reasons are similar to previous studies. In our study the percentage of primiparous women who preferred cesarean section to avoid labor pain were (13.3\%), fear on baby (11.3\%) and fear on herself $(0.7 \%)$. As compared to Dilek Bilgic et al. study the percentage were $(73.3 \%)$ for avoid labor pain, $(53.3 \%)$ for fear on baby and $(60 \%)$ fear on herself. ${ }^{[17]}$

In our study no association was found between preferred mode of delivery and level of education. Although all the education levels chose vaginal delivery more than cesarean section. In contrast to a study done in bander Abbas in which those of lower education level chose cesarean section more than others. ${ }^{[20]}$ In our study no association was found between preferred mode of delivery and occupation. Most of the employed showed less tendency toward the cesarean section which is similar to the study conducted in Bandar Abbas. ${ }^{[20]}$

\section{Conclusion}

Majority of primiparous women interviewed in our research preferred the vaginal delivery, while the remaining women preferred the cesarean section due to lack of knowledge or fear from vaginal delivery. Doctor's recommendation and medical advices play a major role in enhancing the awareness toward vaginal delivery

\section{No conflicts of interest}

Source of funding: self

Ethical clearance: was taken from the scientific committee of the Iraqi Ministry of health 


\section{References}

1. JamshidiManesh M, Oskouie F, Jouybary L, Sanagoo A. The process of women's decision making for selection of cesarean delivery. Iran Journal of Nursing. 2009 Feb;21(56):55-67.

2. Belizán JM, Althabe F, Cafferata ML. Health consequences of the increasing caesarean section rates. Epidemiology. (2007); 18:485-6.

3. Yilmaz SD, Bal MD, Beji NK, Uludag S. Women's preferences of method of delivery and influencing factors. Iranian Red Crescent Medical Journal. 2013 Aug;15(8):683.

4. Aslam MF, Gilmour K, Fawdry RD. Who wants a caesarean section? A study of women's personal experience of vaginal and caesarean delivery. Journal of Obstetrics and Gynaecology. 2003 Jan 1;23(4):364-6.

5. Baker PN, Kenny L, editors. Obstetrics by ten teachers. CRC Press; $19^{\text {th }}$ ed, 2011 Mar 25.

6. World Health Organization. Managing complications in pregnancy and childbirth: a guide for midwives and doctors. World Health Organization; 2017.

7. Elixhauser A, Wier L. Statistical Brief 113. Complicating conditions of pregnancy and childbirth. 2008.

8. Stranges E, Wier LM, Elixhauser A. Complicating conditions of vaginal deliveries and cesarean sections, 2009. HCUP Statistical Brief. 2012;131.

9. Ogasawara M S, Ebara T, Yamada Y, Shoji N, Matsuki T, Kano H, et al. Adverse pregnancy and perinatal outcome in patients with recurrent pregnancy loss: Multiple imputation analyses with propensity score adjustment applied to a large-scale birth cohort of the Japan Environment and Children's Study. American Journal of Reproductive Immunology. 2019; 81(1):e13072.

10. Hakobyan M, Dijkman KP, Laroche S, Naulaers G, Rijken M, Steiner K, et al. Outcome of Infants with Therapeutic Hypothermia after Perinatal Asphyxia and Early-Onset Sepsis. Neonatology. 2019; 115(2):127-133.

11. Carter M. Maternal and neonatal outcomes for cesarean delivery at maternal request (CDMR) during hospital stay: a quantitative study. Neggers YH. Gestational Age and Pregnancy Outcomes. Pregnancy and Birth Outcomes. 2018 Jun 13:9.

12. American College of Obstetricians and Gynecologists. ACOG guidelines on premature rupture of membranes. American Family Physician. (2008); 77(2), 245-246.

1. Fathian Z, Sharifirad GH.R., Hasanzade A. Study of the effects of Behavioral Intention Model education on reducing the cesarean rate among pregnant women of Khomeiny- Shahr. Journal of East Medico. (2006); 9(2):123-31.

2. Gambling DR, Douglas MJ, McKay RS. Obstetrics Anesthesia and Uncommon Disorders. New York: Cambridge University Press; (2008).

3. Farrell SA, Allen VM, Baskett TF. Parturition and urinary incontinence in primiparas. Obstetrics \& Gynecology. (2001); 97(3):350-6.

4. MacArthur C, Bick DE, Keighley MR. Faecal incontinence after childbirth. Br J Obstet Gynaecol. (1997);104(1):46-50.4

5. Bilgic D, Pınar S E, Daglar G, Cesur B. The effect of primiparous pregnant women's preferences for mode of delivery on their fear of childbirth and postpartum period. Clinical nursing studies. (2019); 7:98.

6. Varghese S, Singh S, Kour G, Dhar T. Knowledge, attitude and preferences of pregnant women towards mode of delivery in a tertiary care center. International Journal of Research in Medical Sciences. (2016);4(10):4394-4398

7. Akarsu RH, Mucuk S. Turkish women's opinions about cesarean delivery. Pakistan journal of medical sciences. 2014 Nov; 30(6):1308.

8. Moradabadi A S, Alavi A, Yabandeh A P, Eftekhaari T E, Dadipoor

S. Factors involved in selecting the birth type among primiparous women. Journal of education and health promotion. (2018); 7:55

9. Lukasse M, Schroll AM, Ryding EL, Campbell J, Karro H, Kristjansdottir H, et al. Prevalence of emotional, physical and sexual abuse among pregnant women in six European countries. Acta obstetricia et gynecologica Scandinavica. $2014 \mathrm{Jul}$; 93(7):669-77. 